\title{
Facilitation of molecular motion in nanoparticles and development of turn-on photoacoustic bioprobe for in vivo detection of nitric oxide in encephalitis
}

\author{
Ji Qi ${ }^{1 \dagger}$, Leyan Feng ${ }^{2 \dagger}$, Xiaoyan Zhang ${ }^{3}$, Haoke Zhang ${ }^{1}$, Liwen Huang², Yutong Zhou², \\ Zheng Zhou'2, Zheng Zhao', Xingchen Duan ${ }^{3}$, Fei Xu², Ryan T. K. Kwok ${ }^{1,4}$, Jacky W. Y. \\ Lam $^{1,4}$, Dan Ding ${ }^{3}, X_{\text {Xe Xue }}^{2 *}$, \& Ben Zhong Tang ${ }^{1,4,5 *}$
}

${ }^{1}$ Department of Chemistry, The Hong Kong Branch of Chinese National Engineering Research Center for Tissue Restoration and Reconstruction, Institute for Advanced Study, Department of Chemical and Biological Engineering and Institute of Molecular Functional Materials, The Hong Kong University of Science and Technology, Clear Water Bay, Kowloon, Hong Kong, China. ${ }^{2}$ State Key Laboratory of Medicinal Chemical Biology, College of Pharmacy, Nankai University, Haihe Education Park, 38 Tongyan Road, Tianjin 300353, China. ${ }^{3}$ State Key Laboratory of Medicinal Chemical Biology, Key Laboratory of Bioactive Materials, Ministry of Education, and College of Life Sciences, Nankai University, Tianjin 300071, China. ${ }^{4}$ HKUST-Shenzhen Research Institute, No. 9 Yuexing first RD, South Area, Hi-tech Park, Nanshan, Shenzhen 518057, China. ${ }^{5}$ NSFC Centre for Luminescence from Molecular Aggregates, SCUT-HKUST Joint Research Institute, State Key Laboratory of Luminescent Materials and Devices, South China University of Technology, Guangzhou 510640, China. 'These authors contributed equally to this work. *e-mail: xuexue@nankai.edu.cn; tangbenz@ust.hk

Abstract: Nitric oxide (NO) is an important signaling molecule overexpressed in many diseases, thus the development of NO-activatable probes is of vital significance for monitoring related diseases. However, sensitive photoacoustic (PA) probes for detecting NO-associated complicated diseases (e.g., encephalitis), has yet to be developed. Herein, we report a NOactivated PA probe for in vivo detection of encephalitis by tuning the molecular geometry and energy transformation processes. A strong donor-acceptor structure with good conjugation can be obtained after NO treatment, along with the active intramolecular motion, significantly boosting "turn-on" near-infrared PA property. The molecular probe exhibits high specificity and 
sensitivity towards NO over interfering reactive species. Noninvasive in vivo imaging indicates that PA signal lights up in lipopolysaccharide-induced encephalitis with a high signal-tobackground ratio of 15.7. Further studies reveal that the probe is also capable of differentiating encephalitis in different severities, being beneficial for understanding the disease evolution processes and drug screening. This work will inspire more insights into the development of highperforming NO-activated PA probes for advanced diagnosis by making full use of intramolecular motion and energy transformation processes.

As the basic nature of matter, molecular motion plays an important role in determining many fundamental chemical and physical processes. ${ }^{1,2}$ Continued interest in the field of molecular motion has encouraged controlling and using the transduction of molecular motion energy, for example, to suppress or promote the related energy as needed, which would significantly benefit real applications. ${ }^{3,4}$ For example, the intramolecular motions (e.g., rotation, vibration, and twist) of a chromophore in excited state are directly in association with energy transition processes, and our previous researches about aggregation-induced emission (AIE) suggest that manipulation of the excited-state intramolecular motion could tune the fluorescence property in a highly efficient way. ${ }^{5-7}$ Active intramolecular motion can promote the release of excitation energy via nonradiative decay, a process which determines how much absorbed light can be converted to heat and is closely linked to several important biological techniques such as photoacoustic (PA) imaging. ${ }^{8,9}$

PA imaging is an emerging biomedical imaging technique that relies on ultrasound signals generated by thermoelastic expansions of optically-excited tissue or contrast agents. ${ }^{10,11}$ By availing the benefits of optical resolution and acoustic depth of penetration, PA technique 
enables deep tissue imaging capacity with high spatial resolution and real-time monitoring, rendering great promise for clinical translation. ${ }^{12,13}$ Organic PA imaging agents based on small molecules and polymers have captured intense attention as they possess intrinsic merits such as well-defined structure, large-scale production, ease-of-modification, and good biocompatibility. ${ }^{14,15} \mathrm{PA}$ molecular imaging based on these near-infrared (NIR) contrast agents has been explored for cancer detection, staging and treatment guidance. ${ }^{16-19}$ However, it remains a profound challenge to develop high-performing PA probe for accurate, in vivo diagnosis of complicated and deeply located diseases, like brain inflammation. Thus, it is critically important to exploit organic PA probes with high light-acoustic conversion capacity and high signal-tobackground ratio (SBR) to achieve advanced biomedical diagnosis. The PA effect is closely linked to the photophysical transition process, which could be boosted by tuning the molecular structure and making full use of the absorbed photoenergy. ${ }^{20,21}$ For instance, $\mathrm{Pu}$ et al. pioneered the intraparticle photoinduced electron transfer strategy to quench fluorescence and enhance nonradiative deactivation of semiconducting polymers by doping a fullerene derivative as the acceptor material into nanoparticles (NPs). ${ }^{22}$ Nevertheless, the introduction of a second component increases the complexity of the system. On the other hand, simple structures with improved PA generation property would be more practically useful and merit exploration. As mentioned above, the active intramolecular motion of twisted structures can theoretically promote efficient nonradiative decay to release the excitation energy as heat, which represents a new strategy to boost the performance of PA imaging agents. However, most molecular rotors are only active in solution, and exhibit limited intramolecular motion in aggregate forms such as NPs, which are more useful for biological applications. ${ }^{23-25}$ Another issue for current PA imaging is that most reported PA probes are in "always-on" state and lack specificity. PA signals from 
surrounding normal tissue frequently interferes with signal at the site of interest, leading to false diagnostic outcomes. ${ }^{26,27}$ One solution for this problem is the exploration of activatable PA contrast agents with "off-on" signal in response to a specific biomarker, which can substantially improve the SBR and real-time imaging capability in vivo. ${ }^{28-30}$

Nitric oxide (NO) is an extremely important signaling molecule that plays key roles in regulating various physiological and pathological processes. ${ }^{31,32}$ Studies have shown that NO is overexpressed in many diseases including endothelial dysfunction, cancer, neurodegenerative diseases, and cerebral infection. ${ }^{33-35}$ For instance, the NO concentration in encephalitis site is usually far higher than normal brain tissue. More importantly, NO level could also reflect encephalitis in different stages. ${ }^{36}$ Therefore, the detection of $\mathrm{NO}$ and related diseases is of critical significance for understanding disease severity and progression. Current approaches for detecting $\mathrm{NO}$ are inadequate for in vivo applications, especially real-time noninvasive monitoring of brain diseases. The most widely used method for analyzing NO is the colorimetric Griess assay, which requires acidic conditions and thus has limited in vivo applications. ${ }^{37,38}$ Fluorescence imaging has been developed as a popular method for probing NO with high sensitivity, yet it faces the drawback of shallow penetration depth, which restricts its use to the cellular level and skin surfaces. ${ }^{39-41}$ Clinically used methods such as magnetic resonance imaging could realize nearly unlimited penetration depth. However, their low sensitivity and spatial resolution reduce the detection efficiency. ${ }^{42}$ Owning to its intrinsic advantages, PA imaging represents a promising method to realize real-time detection of NO with high spatiotemporal resolution in vivo. However, it is challenging to develop highly specific and sensitive NO-activated PA nanoprobes to enable precise and in vivo diagnosis of NO-related disease in deep tissue, such as encephalitis. 
In this contribution, we developed a sensitive "turn-on" PA probe with optimal energy transition for in vivo detection of NO in encephalitis. We designed and synthesized a conjugated molecule that could react with NO (Figure 1a), which results in a relatively planar structure with strong intramolecular donor-acceptor (D-A) interaction, and thus a new absorption band in NIR region. Moreover, the molecule becomes highly twisted in excited state, which could further facilitate the active intramolecular motion and thus boost PA conversion. As a result, by combining the planarization in ground state and twistification in excited state, a turn-on NO probe with enhancive PA signal is obtained (Figure 1b). The probe exhibits good selectivity and quantitation towards NO, which enable excellent in vitro PA signal output. The probe has also been employed for noninvasive in vivo PA imaging of $\mathrm{NO}$ in encephalitis and differentiating its severity (Figure 1c), greatly expanding the biomedical applications of active molecular motion as well as the applicability of PA imaging in advanced disease diagnosis and precision medicine.

\section{Results}

Design and synthesis of NO probe. As shown in Figure 1a, a molecular probe with reactiontunable D-A interaction and conformation is designed and synthesized. The octyloxy-substituted triphenylamine (OT) is utilized as the donor unit for the strong electron-donating property and increased solubility. The planar thiophene ring $(\mathrm{T})$ is function as both donor and $\pi$-bridge unit, which can further enhance the electron-donating ability and facilitate the intramolecular charge transfer (ICT). The diamine-substituted benzothiadiazole (AB) is selected as the reaction-tunable acceptor core because the o-phenylenediamino group could react with NO to afford a triazole. ${ }^{43,44}$ As a result, a more electron-deficient $5 H$-[1,2,3]triazolo[4',5':4,5]benzo[1,2$c][1,2,5]$ thiadiazole (TB) structure is formed, which enables much stronger D-A interaction and 
ICT effect. The molecular geometries are also expected to change after NO treatment as the steric hindrance between thiophene and the acceptor core differs. Moreover, the long aliphatic chains are designed to retain some flexible space between the conjugated backbones, which would be favorable for intramolecular motions in aggregate state (e.g., NPs). ${ }^{8,45}$ And the twisted phenyl rings of triphenylamine would also benefit the intramolecular motion. Herein, a D-(NO activatable A)-D type probe (OTTAB) was designed and synthesized, and the synthetic route is depicted in Supplementary Scheme 1. The intermediates and final product have been characterized by nuclear magnetic resonance (NMR) and high-resolution mass spectrum (HRMS) (Supplementary Figure 1-14), and the product (OTTTB) after NO treatment has been verified by HRMS (Supplementary Figure 15).

Photophysical properties before and after NO treatment. The absorption spectra of OTTAB and OTTTB in THF are displayed in Figure 2a. Interestingly, the long-wavelength absorption peak shifts from 459 to $686 \mathrm{~nm}$, allowing pronounced turn-on NIR PA signature. It is noted that OTTTB exhibits a high absorption coefficient of $3.16 \times 10^{4} \mathrm{M}^{-1} \mathrm{~cm}^{-1}$, which is due to the planar conjugated core structure and will ensure strong PA generation property. The NIR absorption band of OTTTB can be assigned to the efficient ICT effect of strong D-A interaction. ${ }^{46,47}$ To investigate the intramolecular motion in aggregate states, water was gradually added into the THF solution, and PL spectra were recorded (Figure $2 b, c)$. The PL intensity of OTTAB decreases at first and then increases gradually with water fraction, suggesting a kind of AIE signature. On the contrary, only very weak PL signal can be observed for OTTTB in high water fractions (Figure 2d), which reveals that intramolecular motion is intense in aggregates and the excited-state energy is dissipated through nonradiative decay. ${ }^{48}$ Moreover, the maximal PL of OTTTB red shifts gradually from $851 \mathrm{~nm}$ to $933 \mathrm{~nm}$ as the water fraction increases 
(Supplementary Figure 16), demonstrating typical twisted intramolecular charge transfer (TICT) effect in high polarity environment. Therefore, dark TICT state is formed in aggregates, in which the active intramolecular motion would facilitate the nonradiative relaxation to generate PA signal. ${ }^{49}$ The different photophysical behaviors of OTTAB and OTTTB may be due to the structure alteration.

Density function theory (DFT) calculation was conducted to study the molecular geometries. As shown in Figure 3a, the dihedral angles between the acceptor core and thiophene rings are greatly decreased from $38.5^{\circ}$ and $36.7^{\circ}$ for OTTAB to $2.8^{\circ}$ and $15.9^{\circ}$ for OTTTB, as the steric hindrance between amino groups and thiophene spacer is much higher. The planar structure obtained after NO treatment enables better conjugation and more efficient ICT, and thus new NIR absorption. As a result, the pronounced alteration in molecular structure of NO treatment leads to distinct photophysical energy conversion processes, in which the maximal nonradiative process of OTTTB would boost the turn-on PA property. As illustrated in Figure 3b, the electronic bandgaps of OTTAB and OTTTB are 2.57 and $1.67 \mathrm{eV}$, respectively. The highest occupied molecular orbital (HOMO) energy level increases slightly (from -4.43 to $-4.40 \mathrm{eV}$ ), while the lowest unoccupied molecular orbital (LUMO) energy level decreases a lot (from -1.86 to $-2.73 \mathrm{eV})$. The HOMO and LUMO energy levels of D-A type compound are usually related to the electronic properties of donor and acceptor moieties, respectively. So this result confirms that the electron-withdrawing property of the acceptor core is activated by NO treatment, and strong D-A interaction is realized.

Preparation and characterization of NPs. To endow the hydrophobic compound with good water solubility and biocompatibility, OTTAB was encapsulated into NPs in the assistant of 1,2distearoyl-sn-glycero-3-phosphoethanolamine- $N$-[methoxy-(polyethylene glycol)-2000] (DSPE- 
$\mathrm{PEG}_{2000}$ ) with nanoprecipitation method (Figure 4a). The hydrophobic molecule is self-assembly in the core, and the amphiphilic lipid-PEG polymer is function as the surface layer. It is noted that the long alkyl chain in DSPE-PEG could tangle with the octyl substitutes in OTTAB, which endues the encapsulated NPs with some flexible domains beneficial for free movement. Accordingly, the NPs' PA effect is expected to promote by the active intramolecular motions. Transmission electron microscopy (TEM) measurement (Figure 4b) suggests that the obtained organic NPs are a kind of sphere structure, and dynamic light scattering (DLS) result gives an average diameter of $142 \mathrm{~nm}$. Due to the hydrophobic nature of the organic probe, the encapsulated NPs are very stable in aqueous media, and the diameter nearly doesn't change after storage in ambient condition for two weeks (Supplementary Figure 17), which manifests potential in vivo applications.

The absorption spectra of the NPs before and after NO treatment are presented in Supplementary Figure 18. Interestingly, a new absorption band with maximum at about $690 \mathrm{~nm}$ appears after reacting with NO, matching well with the absorption spectrum of OTTTB (Figure 2a). The NPs possess strong absorption in the spectral region of $600-800 \mathrm{~nm}$, which locates within the excitation range of commercially available PA instrument (680-950 nm). Meanwhile, the color of NPs solution apparently changes from yellow to green (Supplementary Figure 18). The large bathochromic shift of about $230 \mathrm{~nm}$ in absorption spectra is sufficient for eliminating the signal background from pristine probe. We further studied the PL property as it is a competitive channel to nonradiative PA generation pathway. OTTAB NPs exhibit strong emission, while only very weak PL signal can be observed after NO treatment, indicating that the radiative process is greatly suppressed due to the more planar structure and reduced bandgap (Figure 4c), being favorable for nonradiative decay and PA generation. It is noted that the 
maximal PL of NO-treated OTTAB NPs is at $902 \mathrm{~nm}$, which suggests the formation of dark TICT state, and strong intramolecular motions promoting nonradiative decay within the NPs. To verify the specificity of the probe for sensing NO, we next determined the detection selectivity. A lot of possible reactive species including metal ions, amino acids, and reactive oxygen/nitrogen/sulfur species (ROS/RNS/RSS) have been screened (Figure 4d). Of note, the probe is inert to other ROS, RNS and RSS, such as $\mathrm{H}_{2} \mathrm{O}_{2},{ }^{\circ} \mathrm{OH}, \mathrm{O}^{2-}, \mathrm{ClO}^{-}, \mathrm{SO}_{3}{ }^{2-}, \mathrm{H}_{2} \mathrm{~S}, \mathrm{NO}^{3-}$, and $\mathrm{ONOO}^{-50}{ }^{50}$ Moreover, the probe exhibits neither response to metal ions, nor GSH/Cys. By comparison, only NO treatment could generate strong NIR absorption, illustrating the high selectivity of OTTAB NPs. The relationship between the absorption intensity at $690 \mathrm{~nm}$ and NO concentration was further investigated (the inset of Figure 4d), which demonstrates very good linear behavior. The dose-dependent responsivity toward NO suggests that the probe is suitable for quantitative analysis.

In vitro PA response of the nanoprobe. After confirming the photophysical properties, we next studied the PA response of this probe. PA spectrum of the NO-treated OTTAB NPs is shown in Figure 5a, which is in agreement with the absorption profile, and reveals that the PA signal indeed comes from the NIR absorption of OTTTB. The PA stability was then evaluated by scanning the NO-treated NPs in a phantom with $1.2 \times 10^{4}$ of laser pulses at $700 \mathrm{~nm}\left(17.5 \mathrm{~mJ} \mathrm{~cm}^{-}\right.$ ${ }^{2}$ laser and $10 \mathrm{~Hz}$ pulse repetition rate). Noteworthy, the probe exhibits pretty stable PA signal as there is negligible change in PA amplitude after exposure to pulsed laser, much better than that of clinically used indocyanine green (Figure 5b). This verifies the superb stability and suitability of the probe for long-term PA imaging. The PA signal outcome was further studied by recording the PA intensity of NO-treated OTTAB NPs in different concentrations, and linear correlation between the PA amplitude and probe concentration is observed (Figure 5c). The NO detection 
capacity of the probe was examined by measuring the PA response with the treatment of different NO concentrations. As displayed in Figure 5d, a good linear relationship is observed in relatively low NO concentrations $(<250 \mathrm{nM})$, while the reaction tends to become saturation when further increasing NO concentration. This reveals that the probe holds potential for in situ PA detection of NO-related diseases in different stages. In vitro cellular viability experiments show that more than $90 \%$ of cells maintain alive after treating with a high concentration of the nanoprobe $(50 \mu \mathrm{M})$ (Supplementary Figure 19), revealing good cell compatibility. Taken together, these results reveal that OTTAB NPs is a superb probe for NO sensing, encouraging more exploration for detecting related severe diseases such as encephalitis in situ.

In vivo PA imaging of encephalitis. Encephalitis, or inflammation of brain tissue, is a kind of serious cerebral disease that can cause headache, fever, mental confusion, seizures and even death. ${ }^{51,52}$ Accurate and high-resolution diagnosis of encephalitis in real time greatly benefits effective treatment and successful recovery from encephalitis. It has been reported that brain macrophages (microglia) and astrocytes would be activated during inflammatory pathologies, and up-regulate the expression of inducible nitric oxide synthases (iNOS). ${ }^{53,54}$ iNOS can catalyze the oxidation of $L$-arginine to $L$-citrulline and generate a large amount of NO, which is considered as an important inflammatory mediator that correlates with severity. However, noninvasive in vivo detection of NO-associated encephalitis remains a challenging task due to the lack of highly sensitive and deep-penetrating imaging technology. We thus investigated the feasibility of our PA nanoprobe for detecting NO in encephalitis in living mice. The murine encephalitis model was built by intracerebroventricular administration of lipopolysaccharide (LPS) into mouse brain. LPS, an endotoxin from the outer membrane of bacteria, is known as a potent trigger of inflammation. ${ }^{55,56}$ It has been demonstrated that LPS induces astrocyte and 
microglia activation, as well as overexpression of iNOS and other pro-inflammatory cytokines in brain. $^{57}$

Herein, the right lateral ventricle of mouse brain was pretreated with LPS, whereas the contralateral hemisphere of brain was treated with saline to serve as a control. OTTAB NPs solution ( $\left.2 \mu \mathrm{L}, 1 \mathrm{mg} \mathrm{mL}^{-1}\right)$ was intracerebroventricularly injected to investigate the feasibility for detecting NO-related encephalitis in vivo. PA imaging of mouse brain with intact skull was performed with $700 \mathrm{~nm}$ excitation. A representative PA image of the whole mouse brain clearly illuminates that only the inflamed site shows light-up PA signal after injecting the nanoprobe, suggesting pronounced turn-on PA property of our probe (Supplementary Figure 20). The PA imaging results are shown in Figure 6a, and corresponding PA intensities of brains at different time points post administration are depicted in Figure 6b. Immediately after NPs administration, both the LPS-injected and saline-injected intervals exhibit negligible PA signal, which indicates that LPS or saline treatment does not generate obvious signal interference. Interestingly, PA intensity of the inflamed right ventricle increases rapidly over time, and reaches to maximum at 8 h. In contrast, PA signal of the saline-treated left ventricle nearly does not change throughout the experiment. Quantitative analysis further illuminates that PA intensities of the inflamed ventricles are about 5-fold and 17.7-fold higher than those of the control left ventricles at $4 \mathrm{~h}$ and $8 \mathrm{~h}$ post administration respectively, indicating that OTTAB NPs could sensitively monitor the endogenously generated $\mathrm{NO}$ within encephalitis in vivo. It is also noted that the PA imaging of encephalitis site exhibits a significantly high SBR of 15.7 at $8 \mathrm{~h}$ post-injection, which can be attributed to the excellent turn-on PA responsivity of OTTAB NPs towards NO.

To further explore the utility of our PA nanoprobe to differentiate encephalitis of different severities, various amounts of LPS $\left(1,10,100,500\right.$, and $\left.1000 \mu \mathrm{g} \mathrm{kg}^{-1}\right)$ were used to provoke 
inflammatory response in mice brain. PA imaging was conducted at $8 \mathrm{~h}$ post administration of OTTAB NPs. Encouragingly, the PA intensities in brains consistently intensify with the injected LPS concentrations, and significantly stronger PA signals are observed from the brains pretreated with higher amounts of LPS, which are probably due to that higher LPS concentration induces severer encephalitis. To verify that the PA signal was a result of the activation of OTTAB NPs by NO generated from LPS-induced encephalitis, brains of mice after different amounts of LPS treatment were collected to analyze the iNOS level. iNOS are not only an important indicator involved in inflammation response but also an enzyme that catalyzes the production of NO in encephalitis. Thus, iNOS level in brain could reflect the disease severity and NO generation directly. As shown in Figure 6e, the iNOS mRNA levels of brain are elevated drastically with the doses of LPS used for treatment. This result correlates well with our PA imaging data, and demonstrates that OTTAB nanoprobe could be used for accurately detecting the NO-associated encephalitis in living mice and differentiating their severities, rendering great promise for understanding disease progression and screening drugs.

\section{Discussion}

In this work, we developed a sensitive NO probe with turn-on PA signature for noninvasive in vivo detection of NO in encephalitis. The molecular structure and photophysical transition processes of OTTAB probe change greatly after NO treatment, which results in a strong NIR absorption band and turn-on PA signal output. Moreover, the twistification in dark TICT state and the flexible alkyl substitutes significantly facilitate the intramolecular motions in NPs, which could further boost PA effect. The probe exhibits good selectivity and sensitivity towards NO over other interfering reactive species. Noninvasive in vivo PA imaging with the probe allows 
for detecting NO in encephalitis in a high-contrast manner. Moreover, the probe is also capable of differentiating encephalitis of different severities, being beneficial for understanding the disease evolution processes and drug screening. This work will attract more insights into the development of highly efficient turn-on PA probe for precise biomedical imaging, rendering great promise for real applications.

\section{Methods}

Characterizations. ${ }^{1} \mathrm{H}(400 \mathrm{MHz})$ and ${ }^{13} \mathrm{C}(100 \mathrm{MHz})$ nuclear magnetic resonance (NMR) spectra were recorded on a Bruker AV 400 spectrometer by using $\mathrm{CDCl}_{3}$ as the solvent. Highresolution mass spectra (HRMS) were carried out on a GCT premier CAB048 mass spectrometer. The absorption spectra were measured using a Shimadzu 2550 UV-vis scanning spectrophotometer. The photoluminescence (PL) measurement was carried out on a Horiba Fluorolog-3 Spectrofluorometer. Dynamic light scattering (DLS) measurement was performed on a Malvern Zetasizer Nano ZS-90. Transmission electron microscope (TEM) images were captured by JEOL JEM-1200EX microscope with an accelerating voltage of $80 \mathrm{kV}$.

Preparation of NPs. $1 \mathrm{mg}$ of OTTAB and $2 \mathrm{mg}$ of DSPE-PEG 2000 were dissolved in $1 \mathrm{~mL}$ of tetrahydrofuran (THF) solution, which was poured into $9 \mathrm{~mL}$ of deionized water. Followed by sonication with a microtip probe sonicator (XL2000, Misonix Incorporated, NY) for 2 min. The residue THF solvent was evaporated by violent stirring the suspension in fume hood overnight, and colloidal solution was obtained and used directly. 
Cytotoxicity study. 3-(4,5-Dimethyl-2-thiazolyl)-2,5-diphenyl-tetrazolium bromide (MTT) assay was used to evaluate the cytotoxicity of OTTAB NPs. Detroit 551 human fibroblast cells were respectively harvested in a logarithmic growth phase and seeded in 96 -well plates $\left(2 \times 10^{4}\right.$ cells per well with $100 \mu \mathrm{L}$ suspension) for $24 \mathrm{~h}$ and grew to $\sim 80 \%$ confluence. Then the culture medium was replaced with $100 \mu \mathrm{L}$ of fresh culture medium containing OTTAB NPs with various concentrations (the concentrations based on OTTAB were: $0 \mu \mathrm{M}, 2 \mu \mathrm{M}, 5 \mu \mathrm{M}, 10 \mu \mathrm{M}$, $20 \mu \mathrm{M}$, and $50 \mu \mathrm{M}$, separately). After incubating for $24 \mathrm{~h}$, the culture medium was removed and the wells were washed three times with PBS, and $100 \mu \mathrm{L}$ of MTT dissolved in serum-free culture medium $\left(0.5 \mathrm{mg} \mathrm{mL}^{-1}\right)$ was added into each well. After $4 \mathrm{~h}$, the MTT solution was removed cautiously and $100 \mu \mathrm{L}$ of DMSO was added into the wells, followed by gently shaking for 10 min. Then, the absorbance of MTT was measured by a Bio-Rad 680 microplate reader at $490 \mathrm{~nm}$ to evaluate the viability of cells inside.

Cell culture and stimulation of lipopolysaccharide. Mouse microglial BV2 cell lines were grown in high glucose Dulbecco's modified Eagle's medium (DMEM) supplemented with 10\% fetal bovine serum (FBS), $100 \mathrm{IU} \mathrm{mL}^{-1}$ penicillin, and $10 \mu \mathrm{g} \mathrm{mL}^{-1}$ streptomycin. The cells were maintained in a humidified incubator with $95 \%$ air and a $5 \% \mathrm{CO}_{2}$ atmosphere at $37{ }^{\circ} \mathrm{C}$. The medium containing appropriate agents was replaced every other day. The BV2 cells were treated with LPS (100 ng mL $\mathrm{mL}^{-1}$, and incubated for $24 \mathrm{~h}$. Then the medium is collected for further experiment.

Animals experiments. All animal studies were conducted under the guidelines set by Tianjin Committee of Use and Care of Laboratory Animals, and the overall project protocols were approved by the Animal Ethics Committee of Nankai University. 
Encephalitis model. The Kunming male mice were used for the following experiments. In short, the mice were first anesthetized with 3.5\% isoflurane through intraperitoneal injection. Then the mice were fixed on a brain stereotaxic apparatus (Stoelting, 51500D, USA). The scalp was cut and a hole was drilled at certain position on the skull $(1.0 \mathrm{~mm}$ lateral, $0.2 \mathrm{~mm}$ posterior from bregma). A $5 \mu \mathrm{L}$ of Hamilton syringe with a needle was then inserted through the hole into the lateral ventricle at $2.5 \mathrm{~mm}$ below the horizontal plane of bregma. Different concentrations of LPS in saline and saline $(2 \mu \mathrm{L})$ were infused at the right and left lateral ventricles, respectively.

In vivo PA imaging. OTTAB NPs $\left(2 \mu \mathrm{L}, 1 \mathrm{mg} \mathrm{mL}^{-1}\right)$ were intracerebroventricularly administrated. Then, the scalp was sewed and the mice were anesthetized using $2 \%$ isoflurane in oxygen. In vivo PA imaging of the mouse brain was performed in particular time intervals. The PA imaging was carried out on a commercial small-animal opt-acoustic tomography system (MOST, iThera Medical, Germany). A wavelength-tunable (680-950 nm) optical parametric oscillator (OPO) pumped by a Nd:YAG laser provides excitation pulses with a duration of $7 \mathrm{~ns}$ at a repetition rate of $10 \mathrm{~Hz}$. The light from the fiber covers an area of approximately $4 \mathrm{~cm}^{2}$ with a maximum incident pulse energy of approximately $70 \mathrm{~mJ}$ at $700 \mathrm{~nm}(100 \mathrm{~mJ}, 70 \%$ fiber coupling efficiency). This generates an optical fluence of $17.5 \mathrm{~mJ} \mathrm{~cm}^{-1}$, which is well within the safe exposures according to the American National Standard for Safe Use of Lasers. And PA imaging was performed at $700 \mathrm{~nm}$ excitation. The images then were reconstructed using the model-based algorithm supplied within the View MSOT software suite (V3.6, iThera Medical).

\section{RNA isolation and quantitative reverse transcription polymerase chain reaction. After} perfusion of the heart with ice-cold PBS, the mouse brain was collected. The cortex then was isolated and grinded in $1 \mathrm{~mL}$ of TRIzol on ice, and RNA was extracted using TRIzol reagent following the protocols supplied by the manufacturer. One microgram of total RNA was reverse- 
transcribed into cDNA using PrimeScript RT Master Mix. Then, real-time PCR was conducted using SYBR ${ }^{\circledR}$ GREEN following the manufacturer's instructions. The following primer sets were used: iNOS, forward, 5'-CGT AGC AAA CCA CCA AGT-3'; reverse, 5'-GGT ATG AGA TAG CAA ATC GG-3'; GAPDH, forward, 5'-TTC TCA GCC CAA CAA TAC A-3'; reverse, 5'CCT TGT GGT GAA GAG TGT-3'. The housekeeping gene GAPDH was used for normalization. qPCR was conducted in triplicate for each sample, and target mRNA levels were quantified using the $2-\Delta \Delta \mathrm{CT}$ method.

Data analysis. Quantitative data were expressed as mean \pm standard deviation (SD). Statistical comparisons were made by unpaired Student's $t$-test. $P$ value $<0.05$ was considered statistically significant. All statistical calculations were carried out with GraphPad Prism, including assumptions of tests used (GraphPad Software).

Data availability. All the data supporting the findings in this study are available in the paper and Supplementary Information. Additional data related to this paper are available from the corresponding authors upon request.

\section{References}

1. Gao, M. et al. Mapping molecular motions leading to charge delocalization with ultrabright electrons. Nature 496, 343-346 (2013).

2. Danowski, W. et al. Unidirectional rotary motion in a metal-organic framework. Nat. Nanotechnol. 14, 488-494 (2019). 
3. Ragazzon, G., Baroncini, M., Silvi, S., Venturi, M. \& Credi, A. Light-powered autonomous and directional molecular motion of a dissipative self-assembling system. Nat. Nanotechnol. 10, 70-75 (2015).

4. Dong, J. et al. Ultrathin two-dimensional porous organic nanosheets with molecular rotors for chemical sensing. Nat. Commun. 8, 1142 (2017).

5. Qi, J. et al. Boosting fluorescence-photoacoustic-Raman properties in one fluorophore for precise cancer surgery. Chem 5, 2657-2677 (2019).

6. Hong, Y., Lam, J. W. Y. \& Tang, B. Z. Aggregation-induced emission. Chem. Soc. Rev. 40, 5361-5388 (2011).

7. Mei, J., Leung, N. L. C., Kwok, R. T. K., Lam, J. W. Y. \& Tang, B. Z. Aggregation-induced emission: together we shine, united we soar! Chem. Rev. 115, 11718-11940 (2015).

8. Zhao, Z. et al. Highly efficient photothermal nanoagent achieved by harvesting energy via excited-state intramolecular motion within nanoparticles. Nat. Commun. 10, 768 (2019).

9. Gao, W. et al. sulfide nanoparticles as a photothermal switch for TRPV1 signaling to attenuate atherosclerosis. Nat. Commun. 9, 231 (2018).

10. Wang, L. V. \& Hu, S. Photoacoustic tomography: in vivo imaging from organelles to organs. Science 335, 1458-1462 (2012).

11. Weber, J., Beard, P. C. \& Bohndiek, S. E. Contrast agents for molecular photoacoustic imaging. Nat. Med. 13, 636-650 (2016). 
12. $\mathrm{Pu}, \mathrm{K}$. et al. Semiconducting polymer nanoparticles as photoacoustic molecular imaging probes in living mice. Nat. Nanotechnol. 9, 233-239 (2014).

13. Moore, C. et al. Photoacoustic imaging for monitoring periodontal health: A first human study. Photoacoustics 12, 67-74 (2018).

14. Qi, J. et al. Highly stable organic small molecular nanoparticles as an advanced and biocompatible phototheranostic agent of tumor in living mice. ACS Nano 11, 7177-7188 (2017).

15. Chen, Z. et al. An optical/photoacoustic dual modality probe: Ratiometric in/ex vivo imaging for stimulated $\mathrm{H}_{2} \mathrm{~S}$ upregulation in mice. J. Am. Chem. Soc. 141, 17973-17977 (2019).

16. Lovell, J. F. et al. Porphysome nanovesicles generated by porphyrin bilayers for use as multimodal biophotonic contrast agents. Nat. Mater. 10, 324-332 (2011).

17. Xiao, H. et al. Ratiometric photoacoustic imaging of endoplasmic reticulum polarity in injured liver tissues of diabetic mice. Chem. Sci. 8, 7025-7030 (2017).

18. Ai X. et al. Multispectral optoacoustic imaging of dynamic redox correlation and pathophysiological progression utilizing upconversion nanoprobes. Nat. Commun. 10, 1087 (2019).

19. Liu, Y., Bhattarai, P., Dai, Z. \& Chen, X. Photothermal therapy and photoacoustic imaging via nanotheranostics in fighting cancer. Chem. Soc. Rev. 48, 2053-2108 (2019).

20. Ng, K. K. \& Zheng, G. Molecular interactions in organic nanoparticles for phototheranostic applications. Chem. Rev. 115, 11012-11042 (2015). 
21. Qi, J. et al. Light-driven transformable optical agent with adaptive functions for boosting cancer surgery outcomes. Nat. Commun. 9, 1848 (2018).

22. Lyu, Y. et al. Intraparticle molecular orbital engineering of semiconducting polymer nanoparticles as amplified theranostics for in vivo photoacoustic imaging and photothermal therapy. ACS Nano 10, 4472-4481 (2016).

23. Kaleta, J. et al. Surface inclusion of unidirectional molecular motors in hexagonal tris(ophenylene)-cyclotriphosphazene. J. Am. Chem. Soc. 139, 10486-10498 (2017).

24. Xi, D. et al. NIR light-driving barrier-free group rotation in nanoparticles with an $88.3 \%$ photothermal conversion efficiency for photothermal therapy. Adv. Mater. 32, 1907855 (2020).

25. Li, X. et al. Biodegradable $\pi$-conjugated oligomer nanoparticles with high photothermal conversion efficiency for cancer theranostics. ACS Nano 13, 12901-12911 (2019).

26. Ohata, J., Bruemmer, K. J. \& Chang, C. J. Activity-based sensing methods for monitoring the reactive carbon species carbon monoxide and formaldehyde in living systems. Acc. Chem. Res. 52, 2841-2848 (2019).

27. Jeevarathinam, A. S., Lemaster, J. E., Chen, F., Zhao, E. \& Jokerst, J. V. Photoacoustic imaging quantifies drug release from nanocarriers via redox chemistry of dye-labeled cargo Angew. Chem. Int. Ed. 59, 4678-4683 (2020).

28. Zhang, J. et al. Activatable photoacoustic nanoprobes for in vivo ratiometric imaging of peroxynitrite. Adv. Mater. 29, 1604764 (2017). 
29. Yang, Z. et al. Activatable semiconducting theranostics: Simultaneous generation and ratiometric photoacoustic imaging of reactive oxygen species in vivo. Adv. Mater. 30, 1707509 (2018).

30. Huang, J. \& Pu, K. Activatable molecular probes for second near-infrared fluorescence, chemiluminescence, and photoacoustic imaging. Angew. Chem. Int. Ed. 10.1002/anie.202001783.

31. Chen, X. et al. Recent progress in the development of fluorescent, luminescent and colorimetric probes for detection of reactive oxygen and nitrogen species. Chem. Soc. Rev. $\mathbf{4 5}$, 2976-3016 (2016).

32. Zhou, E. Y. et al. Near-infrared photoactivatable nitric oxide donors with integrated photoacoustic monitoring. J. Am. Chem. Soc. 140, 11686-11697 (2018).

33. Sun, Y.-Q. et al. A mitochondria-targetable fluorescent probe for dual-channel NO imaging assisted by intracellular cysteine and glutathione. J. Am. Chem. Soc. 136, 12520-12523 (2014).

34. Reinhardt, C. J., Xu, R. \& Chan, J. Nitric oxide imaging in cancer enabled by steric relaxation of a photoacoustic probe platform. Chem. Sci. 11, 1587-1592 (2020).

35. Teng, L. et al. Nitric oxide-activated "dual-key-one-lock" nanoprobe for in vivo molecular imaging and high-specificity cancer therapy. J. Am. Chem. Soc. 141, 13572-13581 (2019).

36. Reinhardt, C. J., Zhou, E. Y., Jorgensen, M. D., Partipilo, G. \& Chan, J. A ratiometric acoustogenic probe for in vivo imaging of endogenous nitric oxide. J. Am. Chem. Soc. 140, 1011-1018 (2018). 
37. Shen, Y., Zhang, Q., Qian, X. \& Yang, Y. Practical assay for nitrite and nitrosothiol as an alternative to the Griess assay or the 2,3-diaminonaphthalene assay. Anal. Chem. 87, 1274-1280 (2015).

38. Zhang, W. et al. situ and real-time imaging of superoxide anion and peroxynitrite elucidating arginase 1 nitration aggravating hepatic ischemia-reperfusion injury. Biomaterials 225, 119499 (2019).

39. Miller, E. W. \& Chang, C. J. Fluorescent probes for nitric oxide and hydrogen peroxide in cell signaling. Curr. Opin. Chem. Biol. 11, 620-625 (2007).

40. Zhang, P. et al. A logic gate-based fluorescent sensor for detecting $\mathrm{H}_{2} \mathrm{~S}$ and $\mathrm{NO}$ in aqueous media and inside live cells. Chem. Commun. 51, 4414-4416 (2015).

41. Tang, J., Guo, Z., Zhang, Y., Bai, B. \& Zhu, W.-H. Rational design of a fast and selective near-infrared fluorescent probe for targeted monitoring of endogenous nitric oxide. Chem. Commun. 53, 10520-10523 (2017).

42. Qi, J. et al. Real-time and high-resolution bioimaging with bright aggregation-induced emission dots in short-wave infrared region. Adv. Mater. 30, 1706856 (2018).

43. Liu, L., Zhang, F., Xu, B. \& Tian, W. Silica nanoparticles based on an AIE-active molecule for ratiometric detection of RNS in vitro. J. Mater. Chem. B 5, 9197-9203 (2017).

44. Hu, W. et al. Nitric oxide activatable photosensitizer accompanying extremely elevated twophoton absorption for efficient fluorescence imaging and photodynamic therapy. Chem. Sci. 9, 999-1005 (2018). 
45. Jin, M., Chung, T. S., Seki, T., Ito, H. \& Garcia-Garibay, M. A. Phosphorescence control mediated by molecular rotation and aurophilic interactions in amphidynamic crystals of 1,4bis[tri-(p-fluorophenyl) phosphane-gold(I)-ethynyl]benzene. J. Am. Chem. Soc. 139, 1811518121 (2017).

46. Qi, J., Qiao, W. \& Wang, Z. Y. Advances in organic near-infrared materials and emerging applications. Chem. Rec. 16, 1531-1548 (2016).

47. Ni, J.-S. et al. Photoinduced nonadiabatic decay-guided molecular motor triggers effective photothermal conversion for hyperthermia cancer therapy. Angew. Chem. Int. Ed. DOI: 10.1002/anie.202002516.

48. Li, Q. \& Li, Z. The strong light-emission materials in the aggregated state: What happens from a single molecule to the collective group. Adv. Sci. 4, 1600484 (2017).

49. Liese, D. \& Haberhauer, G. Rotations in excited ICT states fluorescence and its microenvironmental sensitivity. Isr. J. Chem. 58, 813-826 (2018).

50. Bruemmer, K. J., Crossley, S. W. M. \& Chang, C. J. Activity-based sensing: A synthetic methods approach for selective molecular imaging and beyond. Angew. Chem. Int. Ed. DOI: 10.1002/anie.201909690.

51. Varadkar, S. et al. Rasmussen's encephalitis: clinical features, pathobiology, and treatment advances. Lancet Neurol. 13,195-205 (2014).

52. Liu, S. et al. Constitutional isomerization enables bright NIR-II AIEgen for braininflammation imaging. Adv. Funct. Mater. 30, 1908125 (2020). 
53. Sheng, W. et al. Pro-inflammatory cytokines and lipopolysaccharide induce changes in cell morphology, and upregulation of ERK1/2, iNOS and sPLA2-IIA expression in astrocytes and microglia. J. Neuroinflammation 8, 121(2011).

54. Huo, Y. et al. Aromatic secondary amine-functionalized fluorescent NO probes: improved detection sensitivity for NO and potential applications in cancer immunotherapy studies. Chem. Sci. 10, 145-152 (2019).

55. Catorce, M. N. \& Gevorkian, G. LPS-induced murine neuroinflammation model: Main features and suitability for pre-clinical assessment of nutraceuticals. Curr. Neuropharmacol. 14, 155-164 (2016).

56. Chen, Q. et al. $\mathrm{H}_{2} \mathrm{O}_{2}$-responsive liposomal nanoprobe for photoacoustic inflammation imaging and tumor theranostics via in vivo chromogenic assay. Proc. Natl. Acad. Sci. U.S.A. 114, 5343-5348 (2017).

57. Ryu, K.-Y. et al. Dasatinib regulates LPS-induced microglial and astrocytic neuroinflammatory responses by inhibiting AKT/STAT3 signaling. J. Neuroinflammation 16, 190 (2019).

\section{Acknowledgements}

This work was supported by the National Natural Science Foundation of China (51873092, 51961160730, 21788102, 31922045, 31771031, and 81701829), the National Key R\&D Program of China (Intergovernmental cooperation project, 2017YFE0132200), the National Key Research and Development Program of China (2018YFA0209800), the Research Grants Council of Hong 
Kong (C6009-17G and A-HKUST605/16), the Innovation and Technology Commission (ITCCNERC14SC01 and ITCPD/17-9), the Fundamental Research Funds for the Central Universities, Nankai University, China, and the Science and Technology Plan of Shenzhen (JCYJ20160229205601482, JCYJ20170818113348852 and JCYJ20180507183832744).

\section{Author contributions}

D.D., X.X. and B.Z.T. conceived and designed the study. J.Q. synthesized and characterized the compounds. J.Q., L.F. and X.Z. performed the NP preparation and in vitro experiments. L.F., X.Z. and S.J. performed the in vivo experiments. H.Z. provided technical assistance with theoretical calculation. J.Q., F.L., L.H., Y.Z., Z.Z., Z.Z., X.D., R.T.K.K., J.W.Y.L., D.D., X.X. and B.Z.T. analysed the data and participated in the discussion. J.Q., L.F., D.D., X.X. and B.Z.T. contributed to the writing of this paper.

\section{Additional information}

Supplementary information is available in the online version of the paper. Reprints and permissions information is available online at www.nature.com/reprints. Correspondence and requests for materials should be addressed to X.X. and B.Z.T.

\section{Competing interests}

The authors declare no competing interests. 
a

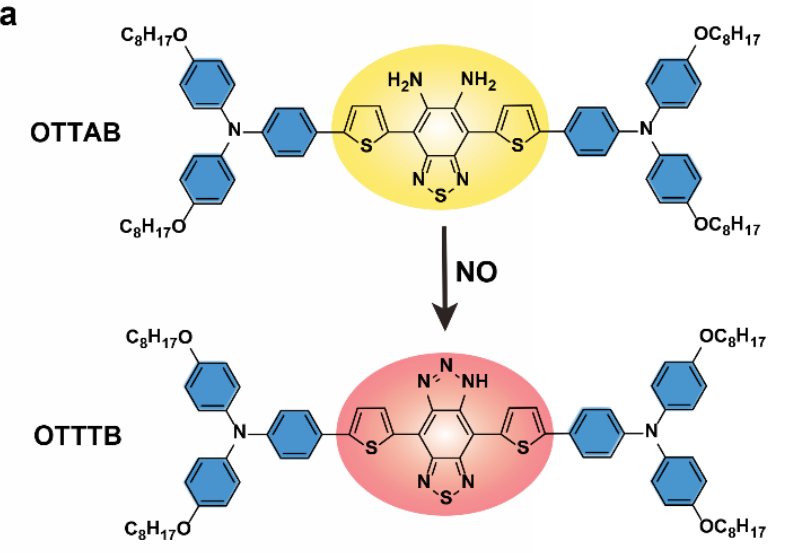

b

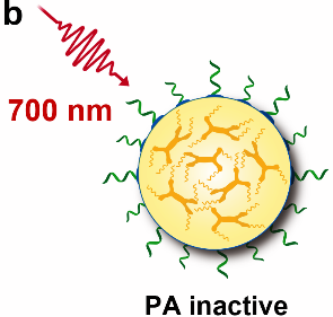

C
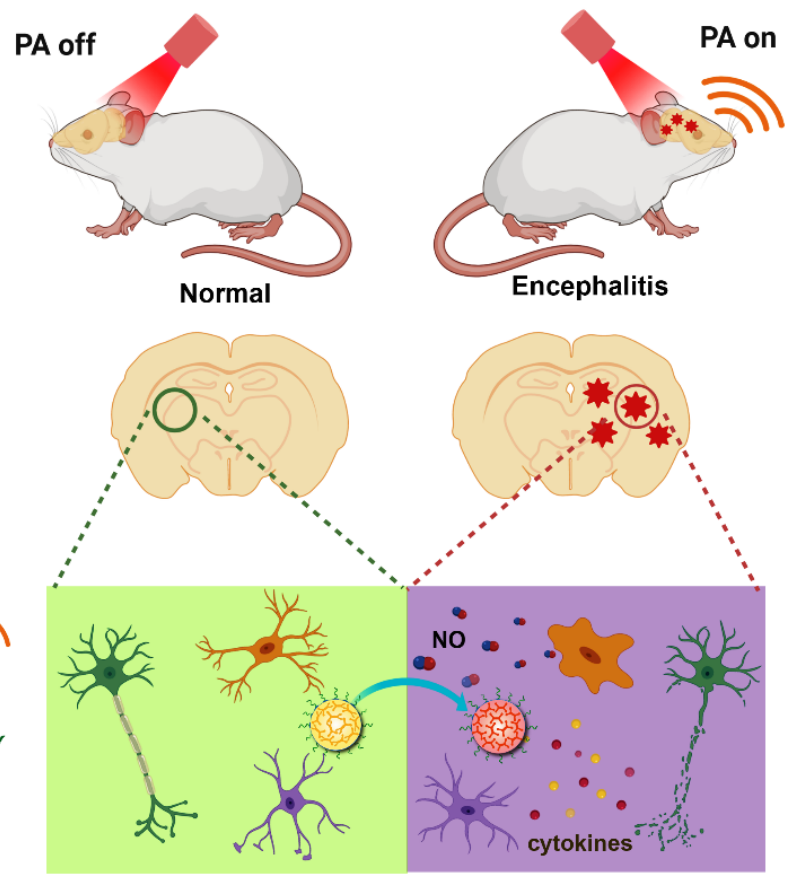

Figure 1 | Turn-on PA probe for NO detection in vivo. a, Changes of chemical structures and b, NPs property after NO treatment. c, Schematic illustration of the in vivo detection of encephalitis with the turn-on PA probe. 

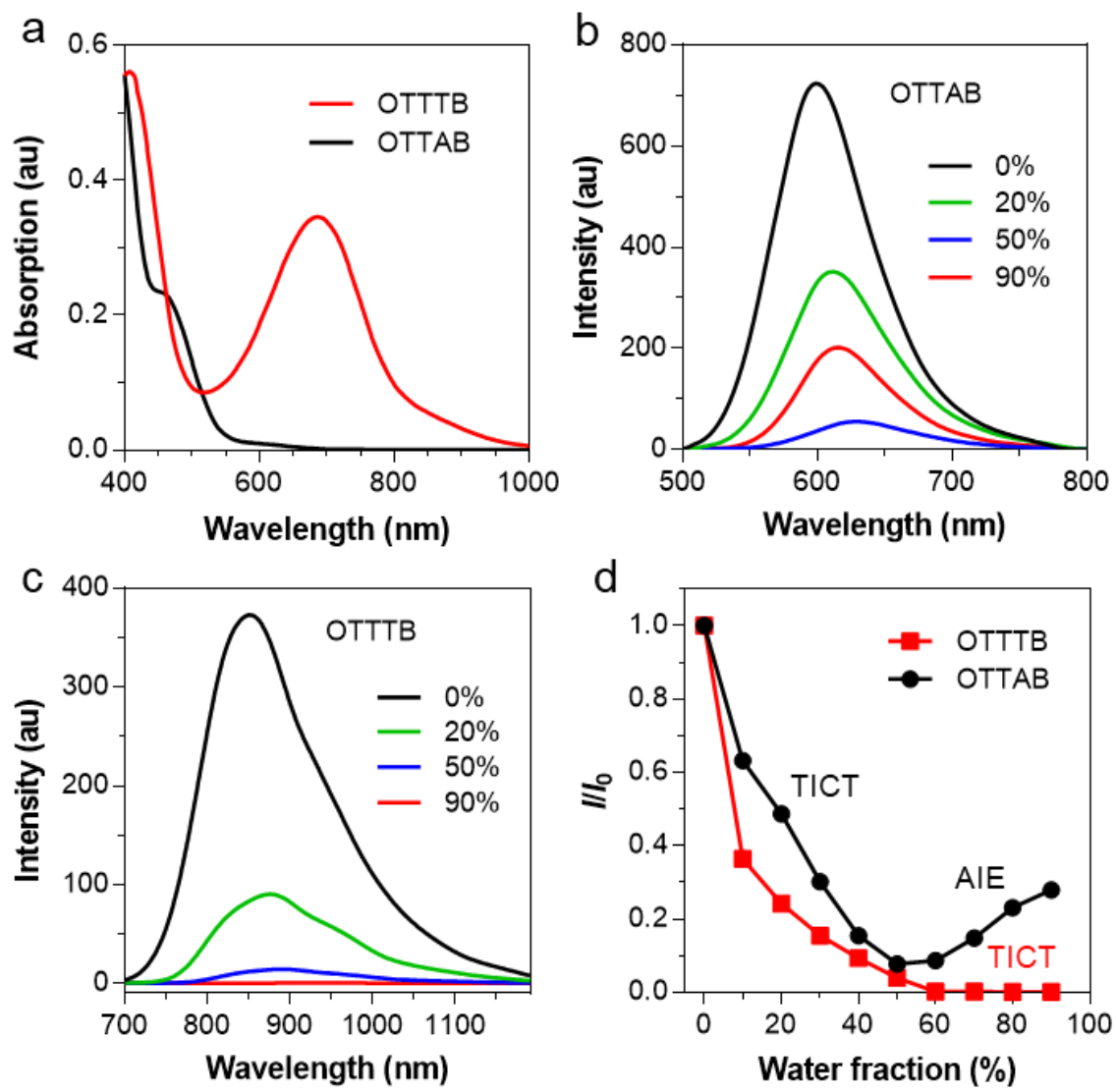

Figure 2 | Photophysical properties before and after NO treatment. a, Absorption spectra of OTTAB and OTTTB in THF $\left(10^{-5} \mathrm{M}\right)$. PL spectra of $\mathbf{b}$, OTTAB and $\mathbf{c}$, OTTTB $\left(10^{-5} \mathrm{M}\right)$ in THF/water mixture with various water fractions $\left(f_{\mathrm{w}}\right)$ as indicated (The excitation wavelengths of OTTAB and OTTTB are 460 and $680 \mathrm{~nm}$, respectively). d, Plot of the PL peak intensity of OTTAB and OTTTB $\left(10^{-5} \mathrm{M}\right)$ versus water fractions in THF/water mixture. $I_{0}$ and $I$ are the PL peak intensities in pure THF $\left(f_{\mathrm{w}}=0\right)$ and THF/water mixtures with specific $f_{\mathrm{w}} \mathrm{s}$, respectively. 


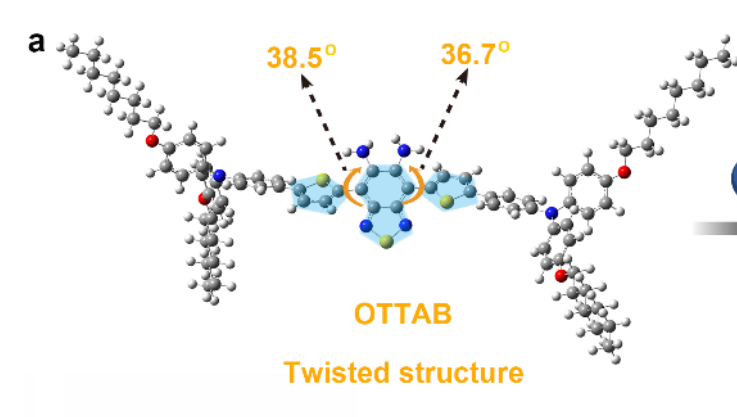

b

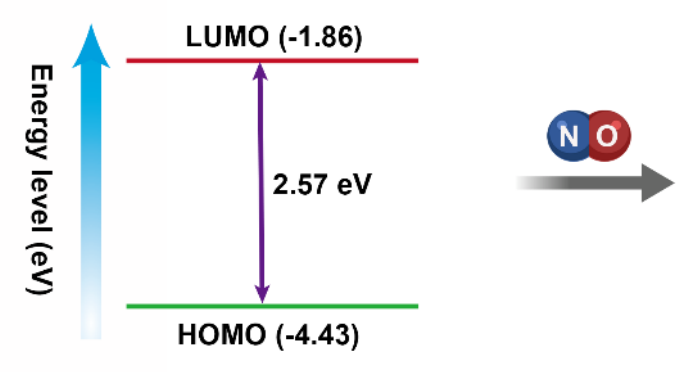

Large bandgap

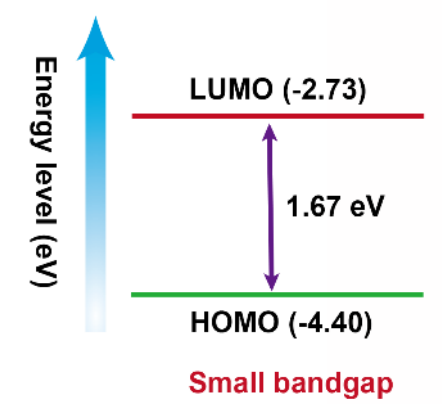

Figure 3 | Changes of molecular conformation and energy level after NO treatment. a, The optimized molecular geometries and $\mathbf{b}$, corresponding changes in the electronic energy levels of OTTAB before and after NO treatment. 

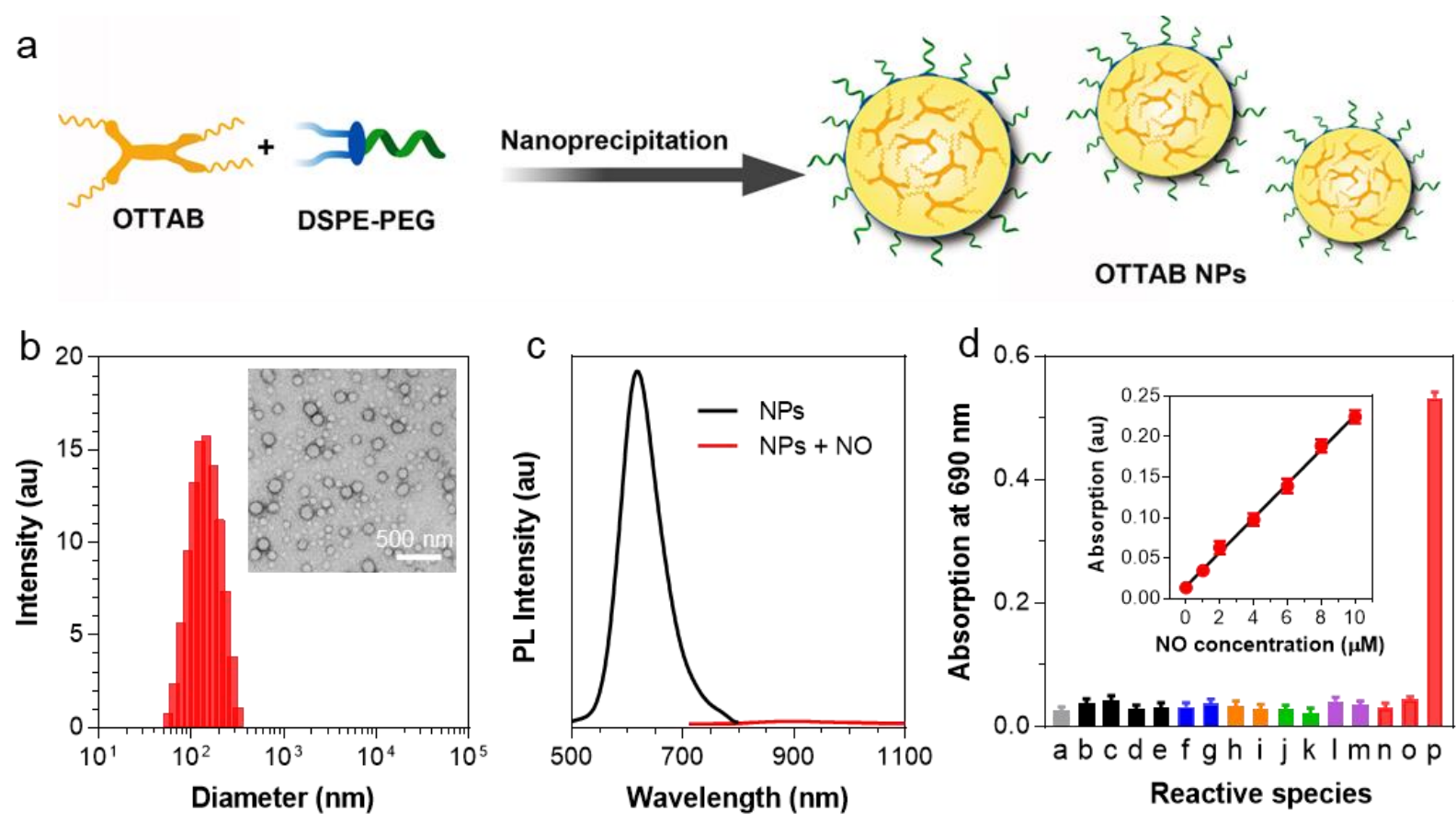

Figure 4 | Characterizations and reactivity of the NPs. a, Schematic illustration showing the nanoprecipitation method. b, Representative DLS and TEM results of the NPs. c, PL spectra of OTTAB NPs $\left(10^{-5} \mathrm{M}\right)$ before and after NO treatment (The excitation wavelengths before and after NO treatment are 460 and $680 \mathrm{~nm}$, respectively). d, Changes of the absorption intensity of OTTAB NPs at $690 \mathrm{~nm}$ with the treatment of different species. a: no treatment, b: $\mathrm{Ca}^{2+}, \mathrm{c}: \mathrm{Fe}^{2+}, \mathrm{d}$ : $\mathrm{Fe}^{3+}$, e: $\mathrm{Cu}^{2+}$, f: GSH, g: $\mathrm{Hcy}, \mathrm{h}: \mathrm{H}_{2} \mathrm{O}_{2}$, i: ${ }^{\circ} \mathrm{OH}, \mathrm{j}: \mathrm{O}^{2-}, \mathrm{k}: \mathrm{ClO}^{-}, \mathrm{l}: \mathrm{SO}_{3}{ }^{2-}, \mathrm{m}: \mathrm{H}_{2} \mathrm{~S}, \mathrm{n}: \mathrm{NO}^{3-}$, o: $\mathrm{ONOO}^{-}, \mathrm{p}$ : NO. Inset shows the relationship between the absorption intensity of OTTAB NPs at $690 \mathrm{~nm}$ and the concentration of added NO. 

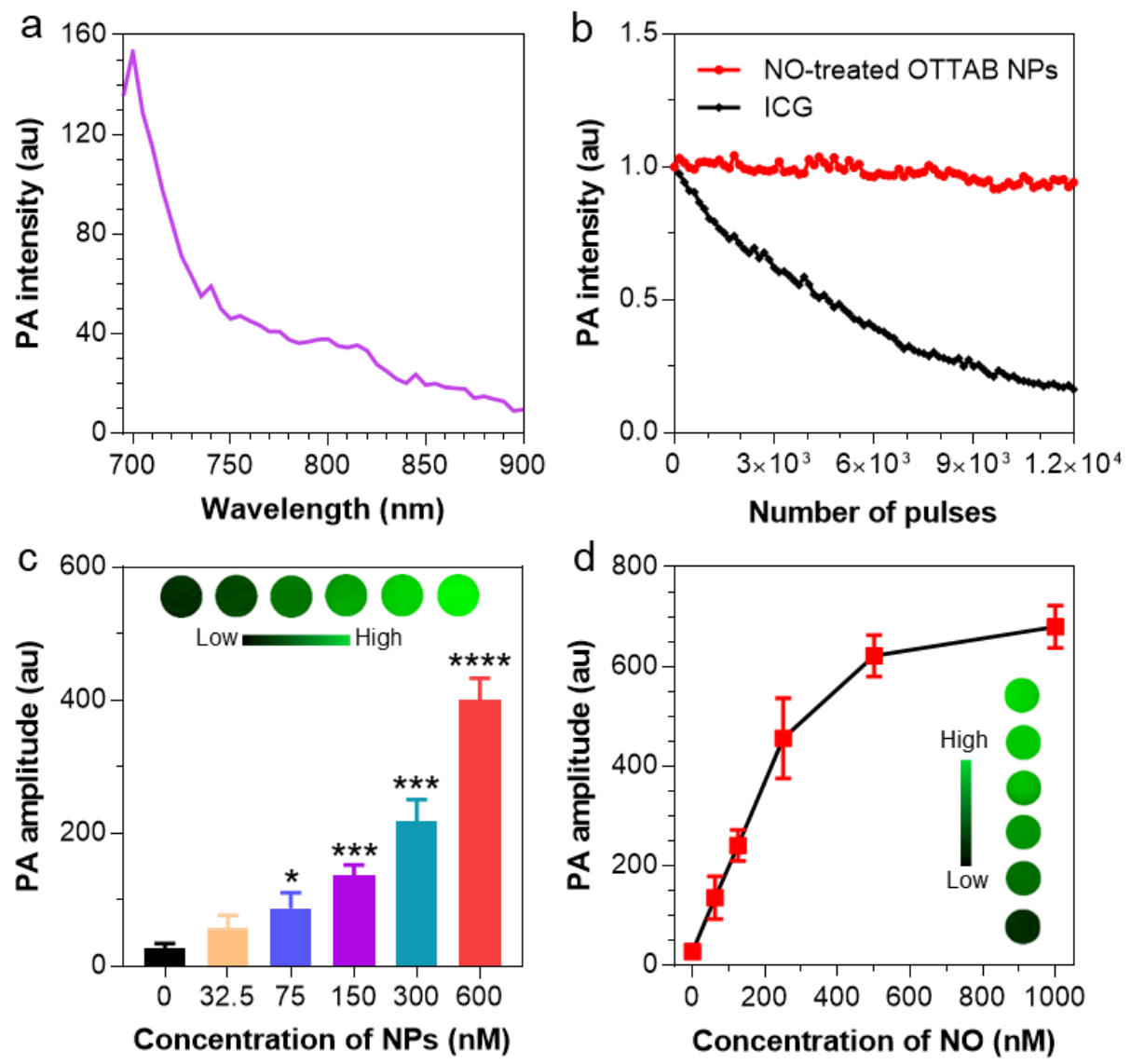

Figure 5 | In vitro detection of NO. a, PA spectrum of the NO-treated OTTAB NPs $(10 \mu \mathrm{M})$. b, Plots of the PA intensity of NO-treated OTTAB NPs $\left(\lambda_{\mathrm{ex}}=690 \mathrm{~nm}\right)$ and indocyanine green (ICG, $\left.\lambda_{\mathrm{ex}}=790 \mathrm{~nm}\right)$ in a phantom $(10 \mu \mathrm{M})$ against number of laser pulses $\left(17.5 \mathrm{~mJ} \mathrm{~cm}^{-2}\right.$ laser and 10 $\mathrm{Hz}$ pulse repetition rate). c, PA amplitudes and the corresponding representative PA images of the NO-treated OTTAB NPs as a function of concentration. Data are presented as the means \pm SD $(n=3)$. d, PA amplitudes and the corresponding representative PA images of OTTAB NPs $(1 \mu \mathrm{M})$ after the treatment with different concentrations of NO. Data are presented as the means $\pm \mathrm{SD}(n=3) . * P<0.05, * * * P<0.001, * * * * P<0.0001$ 

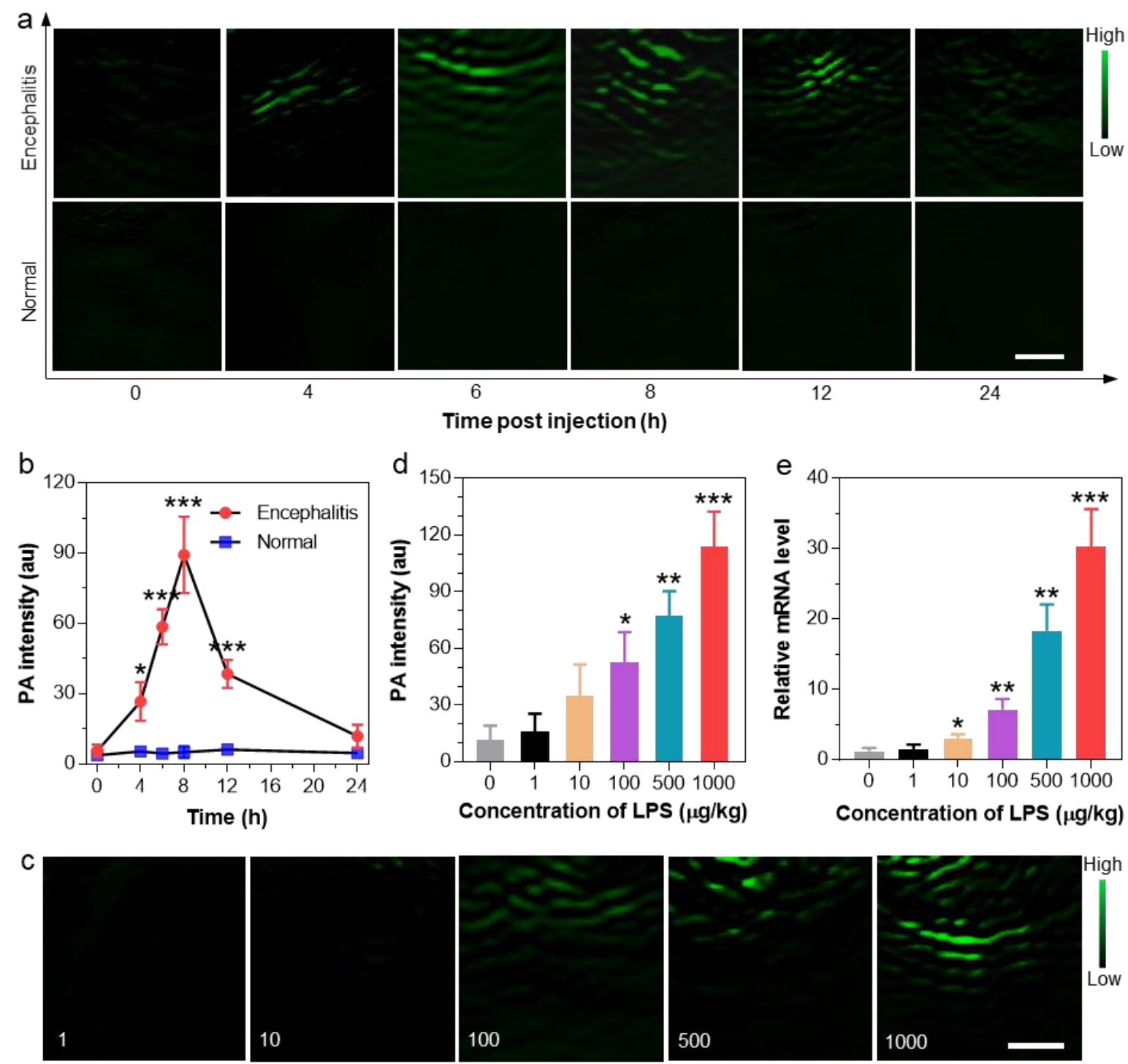

Figure 6 | In vivo PA imaging of encephalitis in different severities. a, Representative in vivo noninvasive PA images and $\mathbf{b}$, the corresponding PA intensity of saline-treated brain (normal) and LPS-induced encephalitis after infusing OTTAB NPs at different time points as indicated. Data are presented as the means \pm SD $(n=4$ mice). c, Representative in vivo noninvasive PA images and d, corresponding PA intensity of the mouse brains treated with different concentrations $\left(\mu \mathrm{g} \mathrm{kg}^{-1}\right)$ of LPS as indicated after administrating OTTAB NPs for $8 \mathrm{~h}$. Data are presented as the means $\pm \mathrm{SD}(n=4$ mice). e, Relative inducible nitric oxide synthase (iNOS) 
mRNA level versus the concentration of injected LPS. Scale bar $=1 \mathrm{~mm} . * P<0.05, * * P<0.01$, $* * * P<0.001$ 